\title{
ThermoKill Database R8110: The Latest Version of a Microbial Thermal Death Database and Its Corresponding Information Statistics
}

\author{
Tomoko $\mathrm{ABE}^{1}$, Jin SAKAMOTO ${ }^{1}$, Tetsuaki TSUCHIDO ${ }^{1,2,}{ }^{\dagger}$, and Ichiro NAKAMURA ${ }^{2,3}$ \\ ${ }^{1}$ Department of Life Science and Biotechnology, Faculty of Chemistry, Material Science and Bioengineering, \\ Kansai University, 3-3-35 Yamate-cho, Suita 564-8680, Japan \\ ${ }^{2}$ TriBioX Laboratories, Ltd., 1-125 Tamaoka-cho, Takano, Sakyo-ku, Kyoto 606-8106, Japan \\ ${ }^{3}$ Department of Child Education, Kyoto Seibo Jogakuin Junior College, \\ 1 Tayamachi, Fukakusa, Fushimi-ku, Kyoto 612-0878, Japan
}

\begin{abstract}
We made a re-expanded version for the database of thermal death of microorganisms, ThermoKill Database (TKDB) R8110. The database was built using data adopted and collected from research papers published between 1981 and 2010 in 25 major selected academic journals. The database includes thermal death data such as 20,467 D values and 3,272 $\mathrm{z}$ values along with predicted $\mathrm{D}$ values at reference temperatures, other microbial information, environmental conditions and bibliographic data for a total of 70 items. Representative statistical distribution data obtained from the novel database were demonstrated and discussed the significance of its practical use in food industry.
\end{abstract}

Key words: thermal death, microorganism, database, information statistics

\section{Introduction}

Systems for assuring microbiological safety of food such as HACCP (hazard analysis critical control point) have recently been developed and widely applied to the manufacturing of different types of food products [i]. Unlike post-processing microbiological tests, the prediction of microbial behavior is of critical importance in the HACCP system. This is especially true for the food heat process where parameters about the microbial heat resistance such as the $D$ value and the $z$ value are required to design a reliable heat process. Here, $D$ value is the decimal reduction time in minutes and $z$ value is the temperature difference (in Celsius) necessary for a decimal reduction in $D$. These parameters are defined by the microbial heat resistance test and then used to construct the $F$ value as process lethality, which is calculated to design and evaluate the heat process.

However, heat resistance of a microorganism varies with the kind of food. Therefore, the predicted values of the above parameters may not be directly applicable to an objective food. In addition, heat resistance varies with not only the heating conditions, but also the type of microorganism and the growth conditions such as the temperature and $\mathrm{pH}$ as pre-heating factors. Meanwhile,

(Received 30 Jul. 2012: accepted 27 Sep. 2012)

† Fax: 06-6388-8609, E-mail: ttsuchi@kansai-u.ac.jp the post-heating factors can affect the ability of a microbial cell to recover from the heat injury during the storage of food and do resultantly its viability [1]. Consequently, it is difficult to determine reliably and satisfactorily the heat resistance and then predict process lethality [2].

One approach to resolve this problem is the building of a sufficiently parameterized database that describes the thermal death behaviors of microorganisms with multiple factors affecting heat resistance. ComBase [ii] includes not only thermal death but also microbial growth and survival during the storage of food and has been used worldwide in food industry. This database also contributes to the construction of the Pathogen Modeling Program (version 7 at present) software for the prediction of the above behaviors of microorganisms. Similar approach has also been taken in another database/predictive software Sym'Previus developed in France [iii]. In Japan, the Japan Food Industry Center has opened their own microbiological safety database [iv]. In these databases, however, only key factors such as the kind of microorganism, temperature, $\mathrm{pH}$ and water activity during the heating period, have been chosen for the thermal death.

We have constructed a literature-based database by collecting thermal death data with many factors as experimental conditions from research articles published in major academic journals since 1996. The first version of 
our database, TKDB R9100, opened in 2000 [3]. The database has since seen versions R8100 [4] and its successor R8105 [5]. In the present paper, we describe the latest version, TKDB R8110. This time, since a further expanded version of the database, TKDB R8110, was completed, we analyzed its novel information statistics on it and introduced its results in this paper.

\section{Materials and Methods}

\subsection{Design of database building}

Data sets of thermal death parameters and conditional factors for growth, heating and recovery of microorganism such as temperature, $\mathrm{pH}$ and the medium composition were taken from research papers published in journals (Fig. 1) and inputted into the file system on the input display image. Since the unit of the input is different for each experimental condition, we defined each input display image as a data set record. The number of items including data and conditions amounts to 70 , which is the same as that in version R8105 [5]. For papers in which $z$ value was not described despite sufficient $D$ value data, we calculated $z$ values using an automatic calculating function and introduced these values into the database via Excel 2007 (Microsoft Corp., Redmond, Washington, U.S.A.). To compare heat resistance between different literatures and between different data sets, a programmed system for the calculation of the reference $D$ values such as $D_{60^{\circ} \mathrm{C}}, D_{121^{\circ} \mathrm{C}}$, using original $D$ value data was applied.

\subsection{Information sources}

We selected 25 academic journals distributed worldwide, as listed in Fig. 1, that published articles on the thermal death of microorganisms. Among those, 19 were English journals and 6 were Japanese.

No. of data record set

$0 \quad 500 \quad 10001500 \quad 2000 \quad 2500 \quad 3000 \quad 3500 \quad 4000 \quad 4500 \quad 5000$

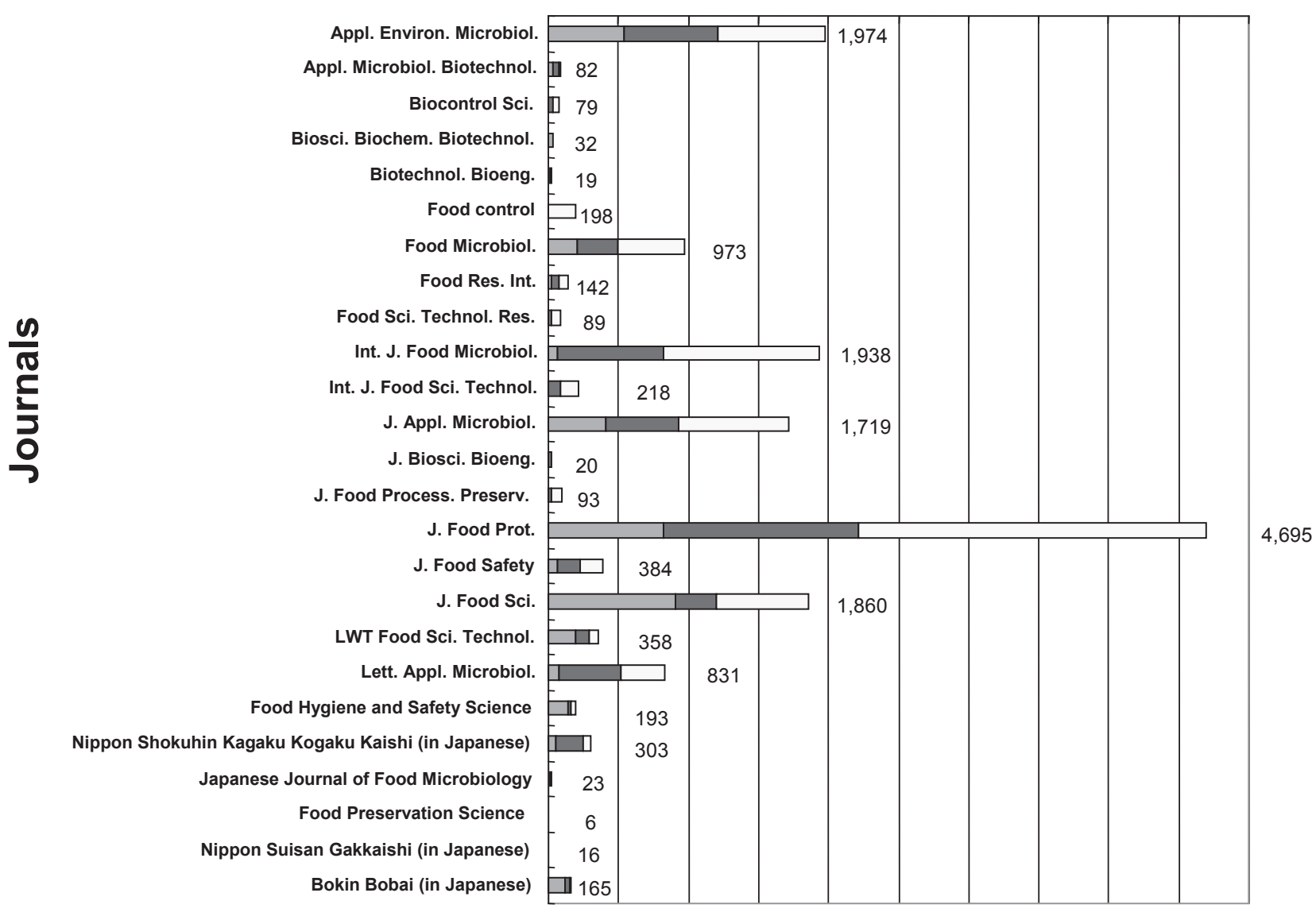

Fig. 1 A histogram of data-set records in TKDB R8110 for journals, from which data were extracted. Grey bar indicates the number of the records obtained from data published in from 1981 to 1990, black bar from 1991 to 2000, and white bar from 2001 to 2010. The number in the figure is a sum total of the records for each journal. 


\subsection{Hardware and software}

For the database format, a formerly designed file based upon FileMaker Pro 7 (FileMaker Inc., Santa Clara, California, U.S.A.) was used on a personal computer (PowerMac G5, Apple Computer Inc. Cupertino, CA, U.S.A.).

\subsection{Retrieving system in the final software product}

The data input system was converted into a retrieving system using File Maker Pro7. For easy retrieving, a popup menu was introduced in major items.

\subsection{Information statistics}

Statistical analysis was done using selected items from the database. To obtain scatter diagrams for the relationship between $z$ values and pH, Excel 2007 was employed.

\section{Results and Discussion}

\subsection{Contents and functions of the novel version of the database}

TKDB R8110 includes 16,410 data record sets, 20,467 $D$ values, 3,272 $z$ values and other experimental factors for a total of 70 items. In comparison with version R8105 [5], TKDB R8110 had 3,493 more records, 3,064 more $D$ values and 560 more $z$ values. These data were extracted from research papers published from the above 25 major journals listed in Fig. 1, which also shows a trend of the number of the record increasing every 10 years. The Journal of Food Protection provides the most data with 4,695 records.

As compared to ComBase, which has basically only four key retrieving items (organism, temperature, $\mathrm{pH}$ and water activity), TKDB R8110 directly provides an individual and substantial information, although it has no predictive function. Like its previous versions $[4,5]$, the database is equipped with an information retrieving system that includes the following search categories, as shown in Fig. 2A. Besides "search by genus and/or species of microorganism", "search by group" is based upon the classification criteria for microorganism such as gram positive/negative and spore former/non-former for bacteria. "Search by combined items" is due to a combination of items selected from microbial genus and/or species and the type for heating menstruum of an objective microorganism. Finally, "search by individual items" can be applied to any arbitrarily selected item among a total of 70 . A search using any of these retrieving catego- ries provides a list of data of major items. Then, each record image is obtained to provide a detailed data set that includes the whole items of bibliographic data, microbiological thermal death data with various environmental and process conditions (Fig. 2B).

\subsection{Statistics of TKDB R8110}

In this latest version, as compared with the previous versions, the number of including data rather increased. Due to this, many more statistical data analyses became possible. Since TKDB R8110 contains many retrieving items like previous versions, different statistical information on each item as well as their statistical relationship with thermal death can be obtained. For example, Fig. 3 shows the statistics of microorganism genera. Based on the number of record sets, the top five bacterial genera were Bacillus (2,541), Listeria $(2,536)$, Escherichia

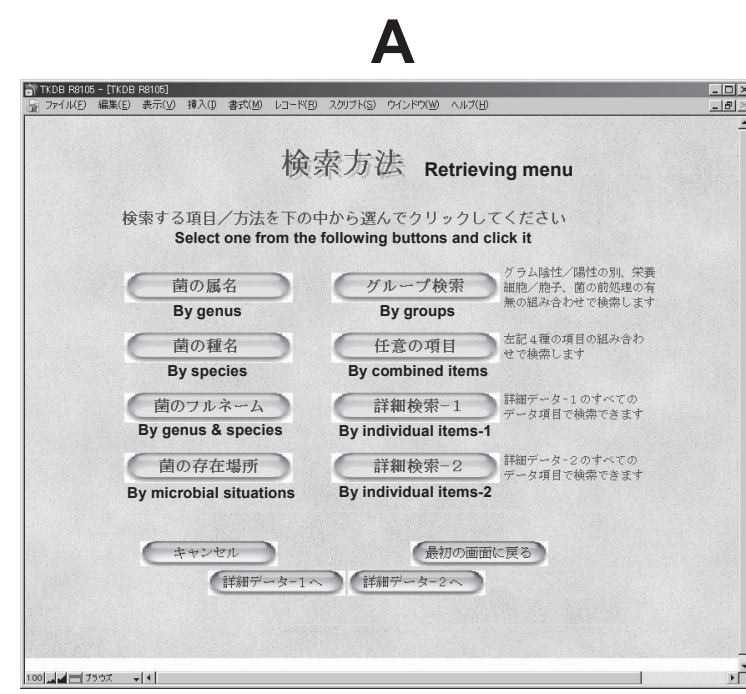

B

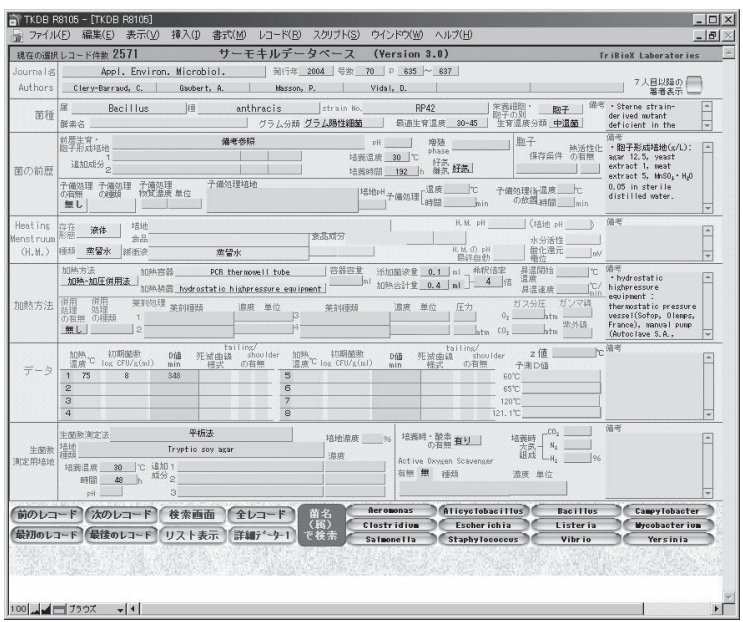

Fig. 2 Personal computer display images of retrieving menu (A). and an example of retrieved result for all items (B). 


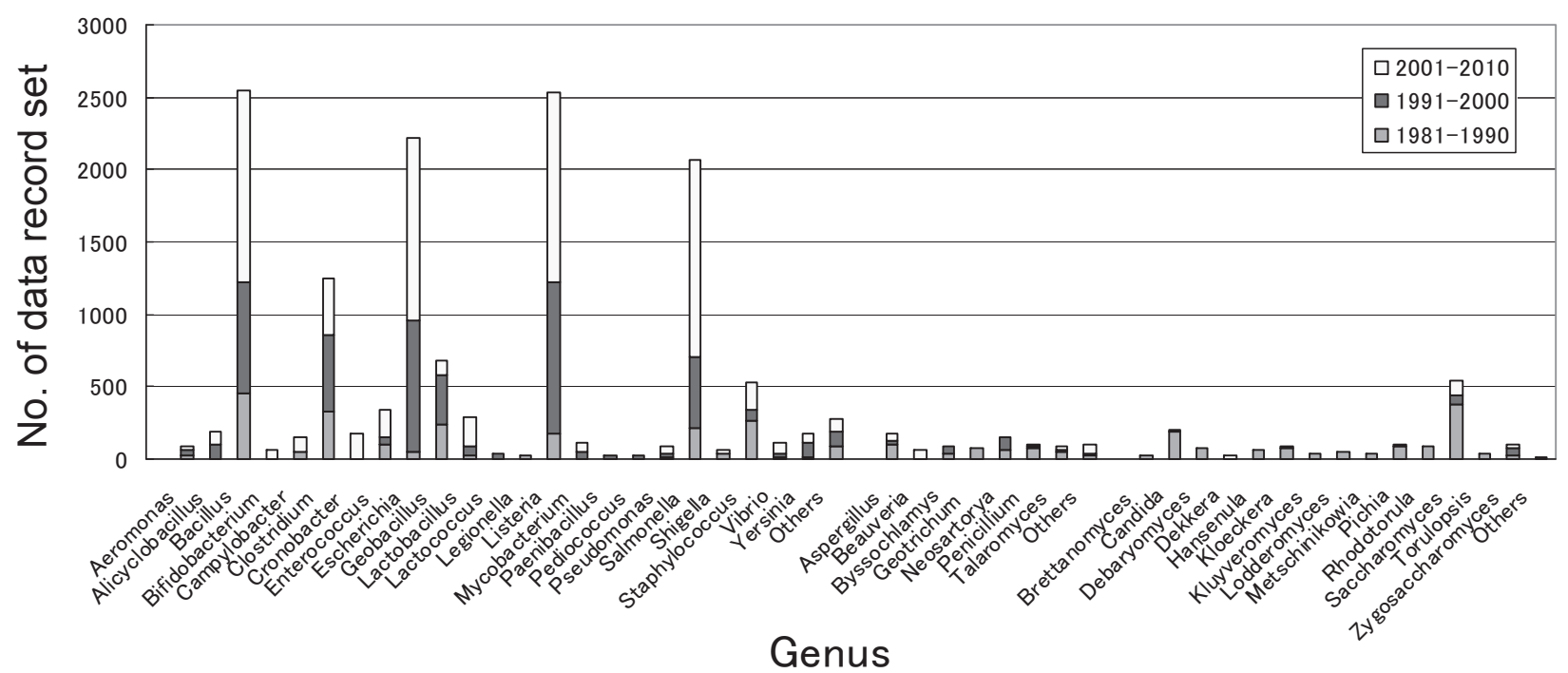

Fig. 3 A histogram of data set records in TKDB ThermoKill Database R8110 for microbial genera.

$(2,214)$, Salmonella $(2,064)$ and Clostridium $(1,244)$, which reflects which microorganisms were concerned over the last 30 years. Additionally, the database shows that bacteria have become increasingly more concerned as a control target than yeasts or molds.

To further examine our database, we investigated the relationship between $z$ values and the pH of heating menstruum for Escherichia. coli and Bacillus cereus spores. The database retrieved 86 and $256 z$ value-pH data sets for these two bacteria, respectively (Fig. 4). Thus, the database can be used to examine how $z$ values distribute with $\mathrm{pH}$ and therefore used to compare these bacteria with an objective microorganism.

As an additional example, we analyzed the relationship between $D_{65^{\circ} \mathrm{C}}$ or $D_{121.1^{\circ} \mathrm{C}}$ at each reference temperature for Escherichia coli $0157: \mathrm{H} 7$ and Bacillus subtilis spores, respectively. The $D$ values were obtained from the original paper or predicted by interpolation or extrapolation using 35 and 58 data sets from each data record for the respective bacterium. For both strains there were many plots at not only neutral but also acidic $\mathrm{pH}$ and the plots distributed over a relatively wide range of $D$ values (Fig. 5).

\subsection{Potential applications}

TKDB R8110 may be applied to evaluate and determine a thermal process. As described previously [3, 4], food manufacturers are able to obtain thermal death data
A

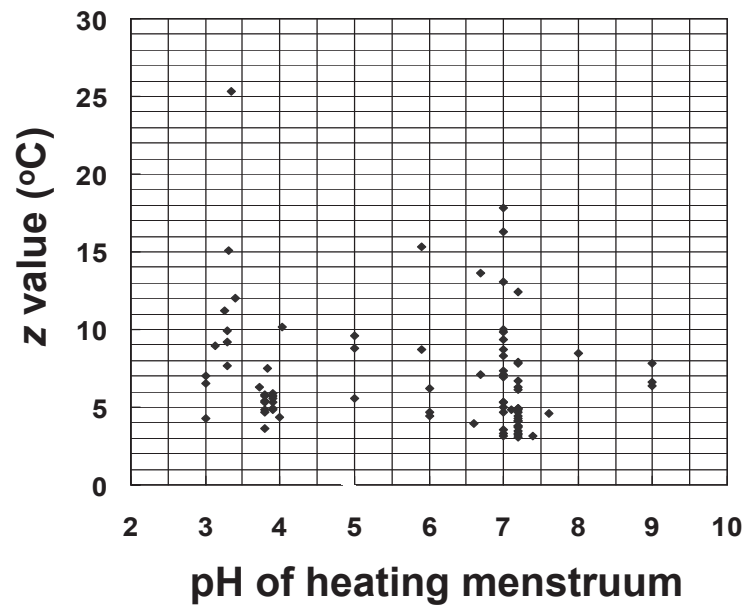

B

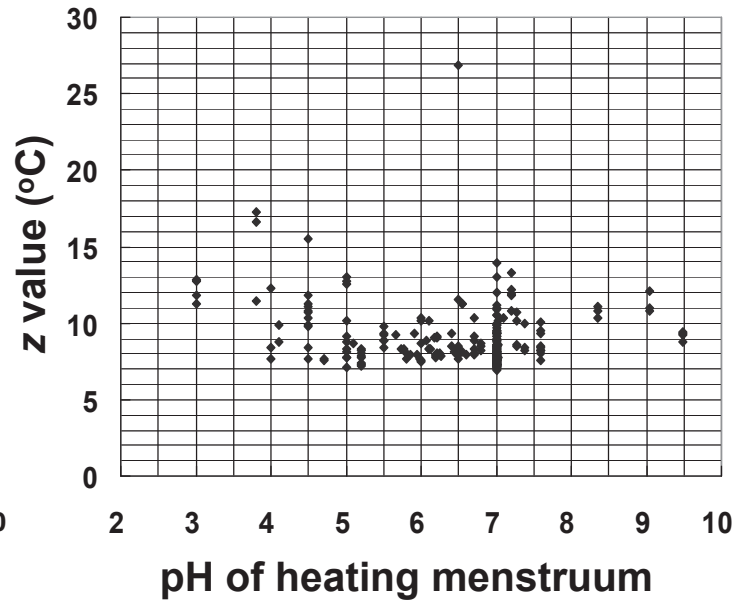

Fig. 4 The relationship between $z$ value and the pH of heating menstruum, which were obtained from data in TKDB R8110. (A) E. coli, (B) B. cereus spores. 
A

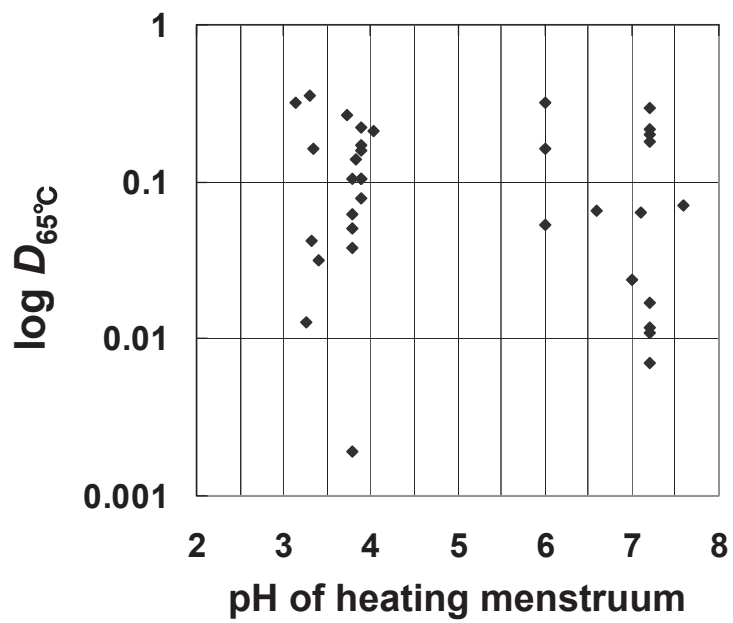

B

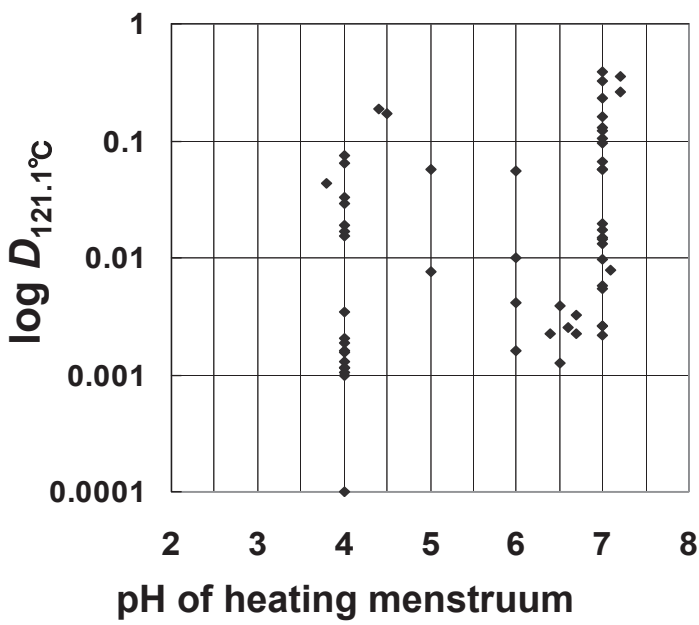

Fig. 5 The relationship between $D$ values at $65^{\circ} \mathrm{C}$ for $E$. coli $\mathrm{O} 157: \mathrm{H} 7$ (A) or at $121.1{ }^{\circ} \mathrm{C}$ for $B$. subtilis spores (B) and the $\mathrm{pH}$ of heating menstruum, which were obtained from data in TKDB R8110.

of an objective or even assumptive target microorganism more easily and rapidly from this and other databases to design an appropriate process and also compare the process with reference data in the database.

In addition, users may collect and survey thermal death data for different parameters that are provided as retrievable items in the database. Such parameters include the kind of microorganism, $\mathrm{pH}$, water activity and the type of heating menstruum, heating method, and the recovery medium for viability assays. The database can also be used to compare thermal death data obtained from a target microorganism with other reference data in the database. For example, Jagannath et al. [6] compared their data of the thermal death of $B$. subtilis pores heated in a buffer at different pHs with data included in ThermoKill Database R9100 for the same species.

\section{Conclusion}

The latest version of our microbial thermal death database, TKDB R8110, has an increased number of $D$ and $z$ values compared to previous versions and also carries data of various conditions and parameters in a style that simplifies retrieving. This version then should be preferred for the determination of the process and evaluation of the sterilization and pasteurization of food.

\section{Acknowledgements}

A part of this study was financially supported by the Japan Food Chemical Research Foundation and the
Iijima Memorial Foundation for Promotion of Food Science and Technology. This study was also supported by the Strategic Project to Support the Foundation of Research Bases at Private Universities, the Ministry of Education, Culture, Sports, Science and Technology in Japan, together with Kansai University.

\section{References}

1) T. Tsuchido; Heat pasteurization and sterilization and their fundamental principles (in Japanese). Clean Technol., 21, (2), 25-29 (2011).

2) T. Tsuchido, I. Nakamura, T. Yokohara; "ThermoKill Database" and its development for predicting food sterilization (in Japanese). J. Antibacterial Antifungal Agents., Japan., 28, 657-662 (2000).

3) I. Nakamura, T. Yokohara, and T. Tsuchido; Database of microbial thermal death. Its construction design based on data from published papers and from experiments performed under defined conditions. Biocontrol Sci., 5, 61-64 (2000).

4) T. Abe, Nakamura, I., T. Yokohara, Y. Omura, T. Hiraga, T. Tsuchido; Construction of ThermoKill Database R8100, an expanded version of a microbial thermal death database developed on the basis of information in research papers published from 1981 to 2000. Biocontrol Sci, 12, 35-38 (2007).

5) T. Abe, T. Tsuchido, I. Nakamura; Development of database software of microbial death caused by heat and chemicals. A novel expanded version of "ThermoKill Database", R8105, and "ChemoKill Database" (in Japanese). Food Ind. (in Japan), 53, (12), 69-74 (2010). 
6) A. Jagannath, I. Nakamura, T. Tsuchido; Modelling the combined effects of $\mathrm{pH}$, temperature and sodium chloride stresses on the thermal inactivation of Bacillus subtilis spores in a buffer system. J. Appl. Microbiol., 95, 135-141 (2003).

\section{URLs cited}

i) U. S. Food and Drug Administration; http://www.fda.gov/
Food/FoodSafety/ HazardAnalysisCriticalControlPointsHAC

CP/default.htm (July 27, 2012)

ii) U. S. Department of Agriculture, Agricultural Research Service; http://www.combase.cc/index.php/ja (July 27, 2012)

iii) Sym'Previus; http://www.symprevius.org (July 27, 2012)

iv) Japan Food Industry Center; http://www.shokusan.or.jp/ haccp (July 27, 2012) 